\title{
A Semilinear Furi-Martelli-Vignoli Spectrum
}

\author{
J. Appell, E. De Pascale and A. Vignoli
}

\begin{abstract}
In this note we extend a spectrum which was introduced by Furi, Martelli and the third author in 1978 for continuous nonlinear maps $F$ to a certain new spectrum for a "semilinear pair" $(L, F)$, with $L$ being a linear Fredholm operator of index zero, and $F$ being nonlinear and continuous.

Keywords: Fredholm operator, continuous nonlinear operator, quasibounded nonlinear operator, measure of non-compactness, $\alpha$-contractive nonlinear operator, nonlinear spectrum, semilinear spectrum, coincidence degree, periodic solution
\end{abstract}

AMS subject classification: 47H12, 47H09, 47H10, 47H11, 47H30, 47A53, 34C25

\section{Nonlinear spectral theory}

Several spectra for nonlinear operators have been studied in the last 30 years. Among them we mention the Kachurovskij spectrum for Lipschitz continuous operators [14] (see also [15]), the Neuberger spectrum for $C^{1}$ operators [18], the Rhodius spectrum for continuous operators [20], and the Dörfner spectrum for linearly bounded operators [5]. All those spectra are modelled on the familiar spectrum for bounded linear operators in Banach spaces and, in fact, reduce to that spectrum in the linear case. As was observed in [4], however, they do not have "good" properties, in general, and they do not lead to natural applications.

On the other hand, in 1978 a nonlinear spectrum was introduced by Furi, Martelli, and Vignoli [12] which is constructed in a completely different way, but has surprisingly many applications, e.g. to nonlinear boundary value problems. The Furi-MartelliVignoli spectrum (or FMV-spectrum, for short) of a continuous nonlinear operator $F$ in a Banach space $X$ is based on solvability properties of the operator equation

$$
F(x)=G(x)
$$

in $X$, where $G$ is a compact operator with a certain asymptotic behaviour. Similarly, Feng [8] defined another spectrum which also builds on solvability properties of equation (1), but now for $G$ satisfying certain boundary conditions on spheres. Here the suitable class is that of stably solvable maps [11] in the case of the FMV-spectrum, and that

J. Appell: Univ. Würzburg, Math. Inst., Am Hubland, D-97074 Würzburg

E. De Pascale: Univ. della Calabria, Dip. di Mat., I-87036 Arcavacata di Rende (CS), Italy

A. Vignoli: Univ. di Roma "Tor Vergata", Dip. di Mat., Via della Ric. Sci., I-00133 Roma appell@mathematik.uni-wuerzburg.de; e.depascale@unical.it; vignoli@mat.uniroma2.it 
of $k$-epi maps [21] in the case of the Feng spectrum. Applying spectral methods to these classes of maps, one gets interesting new existence results which cannot be readily obtained by means of other methods such as topological degree or fixed point theory.

Choosing, in particular, $F(x)=x$ in (1), these existence results reduce to classical fixed point theorems. It is well known that classical fixed point methods apply, whenever one has a problem of the form

$$
L x=F(x)
$$

involving a nonlinear operator $F$ and a linear operator $L$ with trivial null space. Indeed, in this case equation (2) is equivalent to the fixed point problem $x=L^{-1} F(x)$. On the other hand, many boundary value problems for both ordinary and partial differential equations, say, have form (2) with $L$ being a non-invertible linear operator. For this reason Mawhin [16] (see also [13]) replaced the classical Leray-Schauder degree by a certain coincidence degree which was subsequently simplified in [19] and is more adapted to that kind of boundary value problems.

Now, in the recent paper [9], Feng and Webb introduced a spectrum for a pair $(L, F)$, where $L$ is a (not necessarily invertible) linear densely defined Fredholm operator of index zero, and $F$ is a continuous nonlinear operator satisfying some additional requirements. Such a situation arises frequently in applications to differential equations, and the suitable class is that of $(L, k)$-epi maps. In the special case $L=I$, this class reduces to the above mentioned class of $k$-epi maps, and thus the Feng-Webb spectrum reduces to the Feng spectrum described in [8].

The aim of this note is to extend the FMV-spectrum for continuous nonlinear maps $F$ to a certain new spectrum for a "semilinear" pair $(L, F)$, with $L$ and $F$ as above. Loosely speaking, our spectrum is modelled on the FMV-spectrum in a similar way as the Feng-Webb spectrum is modelled on the Feng spectrum. However, our spectrum, denoted by $\sigma(L, F)$, will be defined through the FMV-spectrum of a certain auxiliary map which is simpler than the auxiliary map considered by Feng and Webb.

\section{The FMV-spectrum}

Throughout this paper we will suppose that the following hypotheses are met. $X$ and $Y$ are two Banach spaces over $\mathbb{K}=\mathbb{R}$ or $\mathbb{K}=\mathbb{C}$, and $L: X \rightarrow Y$ is a linear Fredholm operator of index zero. In contrast to the paper [9], we assume in addition that $L$ is bounded; as a matter of fact, this assumption is not really restrictive, since every closed linear operator becomes bounded after passing to a suitable new norm. We have the decompositions $X=N(L) \oplus X_{0}$ and $Y=Y_{0} \oplus R(L)$, where $N(L)$ and $Y_{0}$ have the same (finite) dimension. By $P: X \rightarrow N(L)$ we denote a (bounded) projection on the kernel of $L$, and by $J: N(L) \rightarrow Y_{0}$ a fixed isomorphism between the kernel and cokernel of $L$. By construction, the linear operator $L+J P$ is then invertible between $X$ and $Y$. We mention a typical example which we will consider several times in this paper [17].

Example 1. For fixed $\omega>0$, denote by $C_{\omega}\left[\right.$ resp. $\left.C_{\omega}^{1}\right]$ the space of all continuous [resp. continuously differentiable] $\omega$-periodic functions $x: \mathbb{R} \rightarrow \mathbb{R}^{n}$ with the natural norms

$$
\|x\|_{C_{\omega}}=\max _{0 \leq t \leq \omega}|x(t)|, \quad\|x\|_{C_{\omega}^{1}}=\max _{0 \leq t \leq \omega}|x(t)|+\max _{0 \leq t \leq \omega}\left|x^{\prime}(t)\right| .
$$


Moreover, we write $\widehat{C}_{\omega}$ (resp. $\widehat{C}_{\omega}^{1}$ ) for the subspace of all $x \in C_{\omega}$ (resp. $C_{\omega}^{1}$ ) satisfying

$$
P x:=\frac{1}{\omega} \int_{0}^{\omega} x(t) d t=0 .
$$

The operator $P$ defined by (3) is a continuous projection which maps $C_{\omega}$ onto $\mathbb{R}^{n}$ and so induces a decomposition $C_{\omega}=\widehat{C}_{\omega} \oplus \mathbb{R}^{n}$ resp. $C_{\omega}^{1}=\widehat{C}_{\omega}^{1} \oplus \mathbb{R}^{n}$.

Now, let $X=C_{\omega}^{1}, Y=C_{\omega}$, and define $L: X \rightarrow Y$ by $L x=x^{\prime}$. In the above notation, we have then $N(L)=Y_{0}=\mathbb{R}^{n}, R(L)=\widehat{C}_{\omega}$, and $X_{0}=\widehat{C}_{\omega}^{1}$. So $L$ is a Fredholm operator with $\operatorname{dim} N(L)=\operatorname{codim} R(L)=n$, i.e. of index zero. The bijection $L+J P: C_{\omega}^{1} \rightarrow C_{\omega}$ is given by

$$
(L+J P) x(t)=x^{\prime}(t)+\frac{1}{\omega} \int_{0}^{\omega} x(s) d s
$$

its inverse by

$$
(L+J P)^{-1} y(t)=\int_{0}^{t} y(s) d s-\frac{t}{\omega} \int_{0}^{\omega} y(s) d s+\frac{1}{\omega} \int_{0}^{\omega}\left(1-\frac{\omega}{2}+s\right) y(s) d s .
$$

The nonlinear operator $F$ occurring in equation (2) is very often a Nemytskij operator of the form

$$
F(x)(t)=f(t, x(t))
$$

generated by some Carathéodory function $f:[0, \omega] \times \mathbb{R}^{n} \rightarrow \mathbb{R}^{n}$. For this operator it is possible to calculate the characteristics we are going to study below explicitly [3].

Let us now briefly recall the definition of stably solvable maps. A continuous operator $F: X \rightarrow Y$ is called stably solvable [11] if, for any compact operator $G: X \rightarrow Y$ whose quasinorm

$$
[G]_{Q}=\limsup _{\|x\| \rightarrow \infty} \frac{\|G(x)\|}{\|x\|}
$$

is zero, equation (1) has a solution $x \in X$. Putting, in particular, $G(x) \equiv y$ in this definition, one easily sees that every stably solvable operator $F$ is onto. The converse is true, for example, if $F$ is linear [12].

In what follows, we need two numerical characteristics for nonlinear operators which have also been used in [12]. Apart from the upper quasinorm (5), let

$$
[G]_{q}=\liminf _{\|x\| \rightarrow \infty} \frac{\|G(x)\|}{\|x\|}
$$

denote the lower quasinorm of $F$. Moreover, we will use the two characteristics

$$
[F]_{A}=\inf \{k>0: \alpha(F(M)) \leq k \alpha(M) \text { for any bounded } M \subset X\},
$$

and

$$
[F]_{a}=\sup \{k>0: \alpha(F(M)) \geq k \alpha(M) \text { for any bounded } M \subset X\}
$$


where $\alpha(M)$ denotes the measure of non-compactness of the set $M$ (see, e.g., [1]). Obviously, $F$ is a compact operator if and only if $[F]_{A}=0$.

Recall that the FMV-spectrum [12] of $F: X \rightarrow X$ is defined by

$$
\sigma_{F M V}(F)=\sigma_{q}(F) \cup \sigma_{a}(F) \cup \sigma_{\delta}(F),
$$

where $\lambda \in \sigma_{q}(F)$ if and only if $[\lambda I-F]_{q}=0, \lambda \in \sigma_{a}(F)$ if and only if $[\lambda I-F]_{a}=0$, and

$$
\sigma_{\delta}(F)=\{\lambda \in \mathbb{K}: \lambda I-F \text { is not stably solvable }\} .
$$

In spite of its apparently very technical definition, spectrum (9) has many interesting applications [12]. Moreover, the subspectra in (9) have a simple and natural interpretation in the case of a linear operator $L$ : in fact, $\sigma_{q}(L) \cup \sigma_{a}(L)$ is just the approximate point spectrum of $L$, while $\sigma_{\delta}(L)$ is nothing else but the approximate defect spectrum of $L$.

\section{Definition of the semilinear FMV-spectrum}

Now we are ready to introduce a spectrum for the pair $(L, F)$. To this end, for fixed $\lambda \in \mathbb{K}$ we associate with $(L, F)$ the operator $\Phi_{\lambda}(L, F): X \rightarrow X$ defined by

$$
\Phi_{\lambda}(L, F)(x)=\lambda(I-P) x-(L+J P)^{-1} F(x) .
$$

The operator equation

$$
\Phi_{\lambda}(L, F)(x)=y
$$

is then equivalent to the operator equation

$$
\lambda L x-F(x)=z,
$$

where the equivalence is established by the transform $z=(L+J P) y$. For further reference, we mention the trivial, though useful, equality

$$
\Phi_{\lambda}(L, F)-\Phi_{\mu}(L, F)=(\lambda-\mu)(I-P) \quad(\lambda, \mu \in \mathbb{K}) .
$$

The advantage of the operator $\Phi_{\lambda}(L, F)$ consists in the fact that it acts in one and the same space $X$, while the operator $\lambda L-F$ acts between $X$ and $Y$. Observe, however, that we cannot consider scalar multiples of the identity $I$ in (11), as in classical spectral theory, but of the "perturbed" identity $I-P$. Loosely speaking, one could say that, the "closer" the operator $L$ is to being injective, the "smaller" is its kernel $N(L)$, and hence the "more similar" are the left-hand sides of (12) and (13) to the usual form $\lambda I-N$ with $N$ being some nonlinear operator.

Taking, for example, $X=C_{\omega}^{1}, Y=C_{\omega}$, and $L x=x^{\prime}$ as in Example 1, we end up with the operator $\Phi_{\lambda}(L, F): C_{\omega} \rightarrow C_{\omega}$ given by

$$
\begin{aligned}
\Phi_{\lambda}(L, F)(x)(t)= & \lambda x(t)-\frac{\lambda}{\omega} \int_{0}^{\omega} x(s) d s-\int_{0}^{t} F(x)(s) d s \\
& +\frac{t}{\omega} \int_{0}^{\omega} F(x)(s) d s-\frac{1}{\omega} \int_{0}^{\omega}\left(1-\frac{\omega}{2}+s\right) F(x)(s) d s .
\end{aligned}
$$


Now we are ready to define a semilinear spectrum for the pair $(L, F)$. To this end, we put

$$
\sigma_{\delta}(L, F):=\left\{\lambda \in \mathbb{K}: \Phi_{\lambda}(L, F) \text { is not stably solvable }\right\}
$$

and define the semilinear spectrum of $L$ and $F$ by

$$
\sigma(L, F)=\sigma_{q}(L, F) \cup \sigma_{a}(L, F) \cup \sigma_{\delta}(L, F),
$$

where $\lambda \in \sigma_{q}(L, F)$ if and only if $\left[\Phi_{\lambda}(L, F)\right]_{q}=0$, and $\lambda \in \sigma_{a}(L, F)$ if and only if $\left[\Phi_{\lambda}(L, F)\right]_{a}=0$. Of course, for $L=I$ we simply get $L+J P=I$ and $\Phi_{\lambda}(I, F)=\lambda I-F$, hence

$$
\sigma(I, F)=\sigma_{F M V}(F) .
$$

Thus all results proved below for the semilinear spectrum (16) reduce to a corresponding result for the FMV-spectrum by means of the trivial choice $L=I$. On the other hand, choosing $F=I$ we get the linear operator

$$
\Phi_{\lambda}(L, I)=\lambda(I-P)-(L+J P)^{-1}=(L+J P)^{-1}(\lambda L-I),
$$

and so

$$
\sigma(L, I)=\left\{\frac{1}{\lambda}: \lambda \in \sigma(L) \backslash\{0\}\right\}
$$

consists precisely of the characteristic values of $L$.

\section{Properties of the semilinear FMV-spectrum}

Before proving some properties of spectrum (16), we give a simple lemma which provides several useful connections between the two operators $\Phi_{\lambda}(L, F)$ and $\lambda L-F$.

Lemma 1. Let $\Phi_{\lambda}(L, F)$ be defined as in (11). Then the following equivalences are true:

(a) $\lambda L-F: X \rightarrow Y$ is surjective/injective/bijective if and only if $\Phi_{\lambda}(L, F): X \rightarrow$ $X$ is surjective/injective/bijective, respectively.

(b) $\left[\Phi_{\lambda}(L, F)\right]_{a}>0$ is equivalent to $[\lambda L-F]_{a}>0$.

(c) $\left[\Phi_{\lambda}(L, F)\right]_{q}>0$ is equivalent to $[\lambda L-F]_{q}>0$.

(d) $\Phi_{\lambda}(L, F)$ is stably solvable if and only if $\lambda L-F$ is stably solvable.

Proof. Relations (a), (b) and (c) are immediate consequences of the equivalence of the operator equations (12) and (13). In particular, (b) follows from

$$
\left\|(L+J P)^{-1}\right\|^{-1} \leq[L+J P]_{a} \leq[L+J P]_{A} \leq\|L+J P\|,
$$

and (c) follows from

$$
\left\|(L+J P)^{-1}\right\|^{-1}=[L+J P]_{q} \leq[L+J P]_{Q}=\|L+J P\| .
$$

To prove relation (d) assume first that $\Phi_{\lambda}(L, F)$ is stably solvable, and let $G: X \rightarrow Y$ be compact with $[G]_{Q}=0$. Then the operator $(L+J P)^{-1} G: X \rightarrow X$ is compact 
and satisfies $\left[(L+J P)^{-1} G\right]_{Q}=0$. So by assumption the equation $\Phi_{\lambda}(L, F)(x)=$ $(L+J P)^{-1} G(x)$ has a solution $\hat{x} \in X$. But then $\lambda L \hat{x}-F(\hat{x})=G(\hat{x})$, and so the operator $\lambda L-F$ is stably solvable as claimed.

Conversely, suppose now that $\lambda L-F$ is stably solvable. Let $G: X \rightarrow X$ be compact with $[G]_{Q}=0$. Then the operator $(L+J P) G: X \rightarrow Y$ is compact and satisfies $[(L+J P) G]_{Q}=0$. So again by assumption the equation $\lambda L x-F(x)=(L+J P) G(x)$ has a solution $\hat{x} \in X$. But then $\Phi_{\lambda}(L, F)(\hat{x})=G(\hat{x})$, and so the operator $\Phi_{\lambda}(L, F)$ is stably solvable as claimed

Lemma 1 shows that the definition of spectrum (16) is independent of the operators $P$ and $J$. The following theorem gives a natural topological property of spectrum (16). In the case $L=I$ this theorem reduces to [12: Theorem 8.1.2].

Theorem 1. The spectrum $\sigma(L, F)$ is closed.

Proof. Fix $\lambda \in \mathbb{K} \backslash \sigma(L, F)$, and let

$$
0<\delta<\|I-P\|^{-1} \min \left\{\left[\Phi_{\lambda}(L, F)\right]_{a},\left[\Phi_{\lambda}(L, F)\right]_{q}\right\}
$$

We claim that $\mu \in \mathbb{K} \backslash \sigma(L, F)$ for any $\mu$ satisfying $|\mu-\lambda|<\delta$. First of all, from (14) we get

$$
\left[\Phi_{\mu}(L, F)\right]_{a} \geq\left[\Phi_{\lambda}(L, F)\right]_{a}-|\lambda-\mu|\|I-P\|>0
$$

and

$$
\left[\Phi_{\mu}(L, F)\right]_{q} \geq\left[\Phi_{\lambda}(L, F)\right]_{q}-|\lambda-\mu|\|I-P\|>0,
$$

by our choice of $\delta$. It remains to show that $\Phi_{\mu}(L, F)$ is stably solvable for $|\mu-\lambda|<\delta$. But this follows from [6: Proposition 5], since

$$
\begin{aligned}
\max & \left\{\left[\Phi_{\mu}(L, F)-\Phi_{\lambda}(L, F)\right]_{A},\left[\Phi_{\mu}(L, F)-\Phi_{\lambda}(L, F)\right]_{Q}\right\} \\
& \leq|\mu-\lambda|\|I-P\| \mid \\
& <\min \left\{\left[\Phi_{\lambda}(L, F)\right]_{a},\left[\Phi_{\lambda}(L, F)\right]_{q}\right\} .
\end{aligned}
$$

We conclude that $\lambda$ is an interior point of $\mathbb{K} \backslash \sigma(L, F)$, and so $\mathbb{K} \backslash \sigma(L, F)$ is open

In [12: Proposition 8.1.2/(d)] it was shown that the FMV-spectrum (9) is bounded by $\max \left\{[F]_{A},[F]_{Q}\right\}$, hence compact, provided that the two numbers $[F]_{A}$ and $[F]_{Q}$ are finite, of course. Since $[F]_{A} \leq\|F\|=[F]_{Q}$ for $F$ linear, this gives the usual upper estimate of the spectral radius through the norm in the linear case. However, an analogous result is not true for the semilinear spectrum (16). To see this, it suffices to take $F=I$ and $L$ a linear operator with an unbounded sequence of characteristic values, and to observe that these values belong to the spectrum $\sigma(L, I)$ (see the remark at the end of Section 3). 


\section{Some properties of subspectra}

Let us now take a closer look at the structure of the subspectra $\sigma_{q}(L, F), \sigma_{a}(L, F)$, and $\sigma_{\delta}(L, F)$ defined above. The following result gives some information on the "localization" of these subspectra; in the case $L=I$ it reduces to [12: Proposition 8.1.4].

Theorem 2. The following is true:

(a) $\sigma_{a}(L, F)$ is closed.

(b) $\sigma_{q}(L, F)$ is closed.

(c) $\partial \sigma(L, F) \subseteq \sigma_{a}(L, F) \cup \sigma_{q}(L, F)$.

Proof. (a) Suppose that $\lambda_{n} \in \sigma_{a}(L, F), \lambda_{n} \rightarrow \lambda$, and $\lambda \notin \sigma_{a}(L, F)$. Choose $N \in \mathbb{N}$ such that $\left|\lambda_{n}-\lambda\right|<\left[\Phi_{\lambda}(L, F)\right]_{a}$ for $n \geq N$. Since

$$
\Phi_{\lambda_{n}}(L, F)-\Phi_{\lambda}(L, F)=\left(\lambda_{n}-\lambda\right)(I-P),
$$

we get

$$
\left[\Phi_{\lambda_{n}}(L, F)\right]_{a}=\left[\Phi_{\lambda}(L, F)+\left(\lambda_{n}-\lambda\right)(I-P)\right]_{a} \geq\left[\Phi_{\lambda}(L, F)\right]_{a}-\left|\lambda_{n}-\lambda\right|>0,
$$

contradicting our hypothesis $\lambda_{n} \in \sigma_{a}(L, F)$.

(b) Suppose again that $\lambda_{n} \in \sigma_{q}(L, F), \lambda_{n} \rightarrow \lambda$, and $\lambda \notin \sigma_{q}(L, F)$. Choose $N \in \mathbb{N}$ such that $\left|\lambda_{n}-\lambda\right|<\frac{\left[\Phi_{\lambda}(L, F)\right]_{q}}{\|I-P\|}$ for $n \geq N$. Again by (19) we get then the same contradiction as above.

(c) We show that $\sigma(L, F) \backslash\left[\sigma_{a}(L, F) \cup \sigma_{q}(L, F)\right]$ is open. So fix $\lambda \in \sigma(L, F)$ such that both $\lambda \notin \sigma_{a}(L, F)$ and $\lambda \notin \sigma_{q}(L, F)$. Suppose that there exists a sequence $\left(\lambda_{n}\right)_{n}$ with $\lambda_{n} \rightarrow \lambda$ such that $\Phi_{\lambda_{n}}(L, F)$ is stably solvable. Since $\lambda \in \sigma_{\delta}(L, F)$, there exists a compact operator $G$ of quasinorm zero such that $\Phi_{\lambda}(L, F)(x) \neq G(x)$ for all $x \in X$. On the other hand, by the stable solvability of each $\Phi_{\lambda_{n}}(L, F)$ we find a sequence $\left(x_{n}\right)_{n}$ in $X$ with $\Phi_{\lambda_{n}}(L, F)\left(x_{n}\right)=G\left(x_{n}\right)$. We claim that this sequence $\left(x_{n}\right)_{n}$ is bounded. To see this, suppose that $\left\|x_{n}\right\| \rightarrow \infty$. Then

$$
\begin{aligned}
\frac{\left\|\Phi_{\lambda}(L, F)\left(x_{n}\right)\right\|}{\left\|x_{n}\right\|} & \leq \frac{\left\|\Phi_{\lambda}(L, F)\left(x_{n}\right)-\Phi_{\lambda_{n}}(L, F)\left(x_{n}\right)\right\|}{\left\|x_{n}\right\|}+\frac{\left\|G\left(x_{n}\right)\right\|}{\left\|x_{n}\right\|} \\
& \leq\left|\lambda-\lambda_{n}\right|\|I-P\|+\frac{\left\|G\left(x_{n}\right)\right\|}{\left\|x_{n}\right\|} \\
& \rightarrow 0 \quad(n \rightarrow \infty) .
\end{aligned}
$$

But this means that $\left[\Phi_{\lambda}\right]_{q}=0$, contradicting our choice $\lambda \notin \sigma_{q}(L, F)$. Now, the boundedness of $\left(x_{n}\right)_{n}$ implies that

$$
\left\|\Phi_{\lambda}(L, F)\left(x_{n}\right)-G\left(x_{n}\right)\right\| \leq\left|\lambda_{n}-\lambda\right|\|I-P\|\left\|x_{n}\right\| \rightarrow 0 \quad(n \rightarrow \infty) .
$$

From this and the fact that $\left[\Phi_{\lambda}(L, F)-G\right]_{a}=\left[\Phi_{\lambda}(L, F)\right]_{a}>0$ it follows that there exists a subsequence $\left(x_{n_{k}}\right)_{k}$ of $\left(x_{n}\right)_{n}$ with $x_{n_{k}} \rightarrow \hat{x}$. By continuity, $\hat{x}$ is a solution of the equation $\Phi_{\lambda}(L, F)(\hat{x})=G(\hat{x})$. This contradiction shows that our assumption was false, and so the above assertion is true.

Now, let $\lambda \in \partial \sigma(L, F)$, and assume that $\lambda \notin \sigma_{a}(L, F) \cup \sigma_{q}(L, F)$. Then $\lambda \in$ $\sigma(L, F) \backslash\left[\sigma_{a}(L, F) \cup \sigma_{q}(L, F)\right]$ which is open in $\sigma(L, F)$, contradicting $\lambda \in \partial \sigma(L, F)$ 
In [12] the authors asked whether or not the subspectrum $\sigma_{\delta}(F)$ defined in $(10)$ is also closed, as the other subspectra are. This question is related to the long-standing conjecture that the class of stably solvable maps is stable under small perturbations. Interestingly, this conjecture was disproved quite recently by Furi [10] by means of a sophisticated counterexample involving a retraction of the closed unit ball in the space $X=C[0,1]$ onto its boundary with finite characteristic $(7)$.

If we impose additional conditions on the nonlinear operator $F$, we get more precise information, as we shall see in the next theorem. Given a closed subset $\Sigma$ of the complex plane, by $c_{0}[\Sigma]$ we denote the connected component of $\mathbb{C} \backslash \Sigma$ containing zero, and by $c_{\infty}[\Sigma]$ the unbounded connected component of $\mathbb{C} \backslash \Sigma$.

Theorem 3. Suppose that $X$ and $Y$ are infinite dimensional and $F: X \rightarrow Y$ is compact. Then the following is true:

(a) $\sigma_{a}(L, F)=\{0\}$, hence $\sigma_{a}(L, F) \cup \sigma_{q}(L, F)=\{0\} \cup \sigma_{q}(L, F)$.

(b) $F$ is not onto; in particular, $0 \in \sigma_{\delta}(L, F)$.

(c) Either $0 \in \sigma_{q}(L, F)$, or $c_{0}\left[\sigma_{q}(L, F)\right] \subseteq \sigma_{\delta}(L, F)$.

(d) If $\sigma(L, F) \neq \mathbb{K}$, then $\sigma_{q}(L, F) \neq \emptyset$.

(e) If $0 \notin \sigma_{q}(L, F)$ and $\sigma(L, F)$ is bounded, then $c_{0}\left[\sigma_{q}(L, F)\right]$ is bounded; consequently, $\sigma_{q}(L, F)$ contains a positive and a negative value.

(f) If $\mathbb{K}=\mathbb{C}$ and $\sigma(L, F)$ is bounded, then $c_{\infty}\left[\sigma_{a}(L, F) \cup \sigma_{q}(L, F)\right] \cap \sigma(L, F)=\emptyset$.

Proof. (a) The assertion follows from the fact that, for $F$ compact, the only noncompact part in representation $(11)$ of $\Phi_{\lambda}(L, F)$ is $\lambda I$, and so $\left[\Phi_{\lambda}(L, F)\right]_{a}=|\lambda|$, i.e. $\sigma_{a}(L, F)=\{0\}$.

(b) Being a compact operator between two Banach spaces, $F$ cannot be onto, by Baire's category theorem. Consequently, $\Phi_{0}(L, F)=-(L+J P)^{-1} F$ is not onto either, and thus $0 \in \sigma_{\delta}(L, F)$.

(c) Suppose that $0 \notin \sigma_{q}(L, F)$. This means that $\left[\Phi_{0}(L, F)\right]_{q}>0$. From (a) it follows that 0 is then an isolated point of $\sigma_{a}(L, F) \cup \sigma_{q}(L, F)$. Therefore it suffices to show that $\Phi_{\lambda}(L, F)$ is not surjective for $\lambda$ small enough. In fact, assume that the set $c_{0}\left[\sigma_{q}(L, F)\right] \backslash \sigma_{\delta}(L, F)$ is non-empty. Since this set has no boundary in $c_{0}\left[\sigma_{q}(L, F)\right]$, by Theorem $2 /(\mathrm{c})$, it is both open and closed in $c_{0}\left[\sigma_{q}(L, F)\right]$. But $c_{0}\left[\sigma_{q}(L, F)\right]$ is connected, by definition, and so $\sigma_{\delta}(L, F)=\emptyset$.

Now, to show that $\Phi_{\lambda}(L, F)$ is not surjective for $\lambda$ small enough, assume that this is false. Then there exists a sequence $\left(\lambda_{n}\right)_{n}, \lambda_{n} \rightarrow 0$, such that $\Phi_{\lambda_{n}}(L, F)=$ $\lambda_{n}(I-P)-(L+J P)^{-1} F$ is onto for all $n$. Given $a>0$ with $2 a<\left[\Phi_{0}(L, F)\right]_{q}$, we may find $R>0$ such that $\left\|\Phi_{0}(L, F)(x)\right\| \geq 2 a\|x\|$ for $\|x\| \geq R$. Taking $b:=2 a R$ we have then $\left\|\Phi_{0}(L, F)(x)\right\| \geq 2 a\|x\|-b$ for all $x \in X$.

Fix $y \in X$ with $\|y\| \leq 1$. By assumption, we find a sequence $\left(x_{n}\right)_{n}$ in $X$ such that $\Phi_{\lambda_{n}}(L, F)\left(x_{n}\right)=y$. Without loss of generality we may assume that $\left|\lambda_{n}\right| \leq \frac{a}{\|I-P\|}$ for 
all $n$, where $a$ is as above. Consequently,

$$
\begin{aligned}
1 \geq\|y\| & =\left\|\Phi_{\lambda_{n}}(L, F)\left(x_{n}\right)\right\| \\
& =\left\|\lambda_{n}(I-P) x_{n}+\Phi_{0}(L, F)\left(x_{n}\right)\right\| \\
& \geq 2 a\left\|x_{n}\right\|-b-\left|\lambda_{n}\right|\|I-P\|\left\|x_{n}\right\| \\
& \geq a\left\|x_{n}\right\|-b,
\end{aligned}
$$

hence $\left\|x_{n}\right\| \leq \frac{1+b}{a}$, i.e. the sequence $\left(x_{n}\right)_{n}$ is bounded. We conclude that $\lambda_{n} x_{n} \rightarrow 0$, and thus $F\left(x_{n}\right) \rightarrow-(L+J P) y$ as $n \rightarrow \infty$. Since $y$ with $\|y\| \leq 1$ was arbitrary, we have actually shown that the closure of the set $F\left(\left\{x:\|x\| \leq \frac{1+b}{a}\right\}\right)$ contains a ball (of radius $\left.\left\|(L+J P)^{-1}\right\|^{-1}\right)$, and so has non-empty interior. But this is impossible, because $F$ is a compact operator.

(d) We distinguish the cases $0 \in \sigma_{q}(L, F)$ and $0 \notin \sigma_{q}(L, F)$. In the first case the assertion is trivially true. In the second case it follows from (c) that 0 is an interior point of $\sigma(L, F)$. On the other hand, assertion (a) and Theorem 2/(c) imply that

$$
\partial \sigma(L, F) \subseteq \sigma_{a}(L, F) \cup \sigma_{q}(L, F)=\{0\} \cup \sigma_{q}(L, F)
$$

and $\sigma(L, F)$ has non-empty boundary, since $0 \in \sigma(L, F)$ and $\sigma(L, F) \neq \mathbb{K}$. So we have $0 \in \partial \sigma(L, F)$, which is a contradiction.

(e) If $0 \notin \sigma_{q}(L, F)$, from (c) we conclude that

$$
c_{0}\left[\sigma_{q}(L, F)\right] \subseteq \sigma_{\delta}(L, F) \subseteq \sigma(L, F),
$$

and the assertion follows from the assumed boundedness of $\sigma(L, F)$.

(f) Put $C_{\infty}:=c_{\infty}\left[\sigma_{a}(L, F) \cup \sigma_{q}(L, F)\right]$ and $C:=C_{\infty} \backslash \sigma(L, F)$; we have to show that $C=C_{\infty}$. Since the relative boundary of $C$ with respect to $C_{\infty}$ is empty, by Theorem $2 /(\mathrm{c})$, the set $C$ is both open and closed in $C_{\infty}$. From the connectedness of $C_{\infty}$ it follows that either $C=C_{\infty}$ or $C=\emptyset$. But the latter is impossible if $\sigma(L, F)=C_{\infty} \backslash C$ is bounded

Observe that (f) implies the following alternative on the "size" of the subspectrum $\sigma_{q}(L, F):$ If $\mathbb{K}=\mathbb{C}$, then either $\sigma_{q}(L, F)=\emptyset$ or $0 \in \sigma_{q}(L, F)$ or $\sigma_{q}(L, F)$ is infinite. This is false in the case $\mathbb{K}=\mathbb{R}$. For example, for the operator $F\left(x_{1}, x_{2}, x_{3}, \ldots\right):=$ $\left(\|x\|, x_{1}, x_{2}, \ldots\right)$ in the real sequence space $X=l_{2}$ we have $\sigma_{q}(I, F)=\{ \pm \sqrt{2}\}$.

We illustrate Theorem 3 by a simple example. This example shows, in particular, how both cases in Theorem 3 (c) may occur, and how this gives information on the subspectrum $\sigma_{\delta}(L, F)$ which is most difficult to calculate.

Example 2. Let $X$ be an infinite dimensional complex Banach space, $e \in X$ with $\|e\|=1, L=I$, and

$$
F_{\alpha}(x):=\|x\|^{\alpha} e \quad(\alpha>0) .
$$

It is clear that $\sigma_{a}\left(I, F_{\alpha}\right)=\{0\}$ and $0 \in \sigma_{\delta}\left(I, F_{\alpha}\right)$ for any $\alpha>0$. Concerning $\sigma_{q}\left(I, F_{\alpha}\right)$ and $\sigma_{\delta}\left(I, F_{\alpha}\right)$, we distinguish three cases. 
1st case: $\alpha<1$. Then $\left[F_{\alpha}\right]_{Q}=0$, and so $\sigma\left(I, F_{\alpha}\right)$ can only contain 0 , by the FuriMartelli-Vignoli estimate for the spectral radius. Indeed, $\sigma_{q}\left(I, F_{\alpha}\right)=\{0\}$ since $\left[F_{\alpha}\right]_{Q}=$ 0 , and $\sigma_{\delta}\left(I, F_{\alpha}\right)=\{0\}$ since $F_{\alpha}$ is not onto.

2nd case: $\alpha=1$. This is the most interesting case. Here $\sigma\left(I, F_{1}\right)$ must be contained in the closure of the complex unit disc $\mathbb{D}=\{z \in \mathbb{C}:|z|<1\}$, again by the Furi-MartelliVignoli estimate for the spectral radius. It is easy to see that $\sigma_{q}\left(I, F_{1}\right)=\partial \mathbb{D}$, hence $c_{0}\left[\sigma_{q}\left(I, F_{1}\right)\right]=\mathbb{D}$ and so $\mathbb{D} \subseteq \sigma_{\delta}\left(I, F_{1}\right) \subseteq \overline{\mathbb{D}}$, by Theorem $3 /(\mathrm{c})$. We claim that the operator $\lambda I-F_{1}$ is not onto for $|\lambda|=1$, and so $\sigma_{\delta}\left(I, F_{1}\right)=\overline{\mathbb{D}}$. In fact, the element $e$ does not belong to the range of $\lambda I-F_{1}$ for $|\lambda|=1$. To see this, observe that the equality $\lambda x=\|x\| e+e$ for some $x \in X$ would imply $\|x\|=\|\lambda x\|=\|x\|+1$.

We point out that $c_{\infty}\left[\sigma_{a}\left(I, F_{1}\right) \cup \sigma_{q}\left(I, F_{1}\right)\right]=c_{\infty}[\{0\} \cup \partial \mathbb{D}]=\mathbb{C} \backslash \overline{\mathbb{D}}$, in accordance with Theorem $3 /(\mathrm{f})$.

3rd case: $\alpha>1$. Here the Furi-Martelli-Vignoli estimate does not provide any information on the size of the spectrum, since $\left[F_{\alpha}\right]_{Q}=\infty$ for $\alpha>1$. However, it is easy to see directly that $\sigma_{q}\left(I, F_{\alpha}\right)=\emptyset$, and so $c_{0}\left[\sigma_{q}\left(I, F_{\alpha}\right)\right]=\mathbb{C}$. From Theorem $3 /(\mathrm{c})$ we conclude that $\sigma_{\delta}\left(I, F_{\alpha}\right)=\mathbb{C}$.

We may summarize our results as follows. The spectrum $\sigma\left(I, F_{\alpha}\right)$ consists only of zero if $F_{\alpha}$ has sublinear growth $(\alpha<1)$, coincides with the closed unit disc if $F_{\alpha}$ has linear growth $(\alpha=1)$, and fills the whole complex plane if $F_{\alpha}$ has superlinear growth $(\alpha>1)$. This is of course what one could expect for a reasonable "nonlinear" spectrum.

\section{Operators with discrete spectrum}

Splitting the spectrum of a bounded linear operator into a "discrete" and an "essential" part is important in applications. This decomposition becomes particularly simple for compact linear operators: the non-zero spectrum of such an operator is purely discrete, i.e. consists only of eigenvalues.

In this section we give a parallel result for the semilinear spectrum (16) in the case of a compact nonlinear operator $F$. To this end, we first have to find an appropriate notion of "eigenvalue" which corresponds to this spectrum. It is easy to see (and, in fact, was already observed for the classical FMV-spectrum (9)), that the "naive" definition of an eigenvalue as a scalar $\lambda$ for which the equation $\lambda L x=F(x)$ has a non-trivial solution $x$, is not suitable. For example, for the scalar function $F(x)=\sqrt{|x|}$ in $X=\mathbb{R}$ we have $\sigma(I, F)=\sigma_{F M V}(F)=\{0\}$, but each $\lambda \in \mathbb{R} \backslash\{0\}$ is an eigenvalue of $F$, and so the point spectrum may even be disjoint from the FMV-spectrum.

The following notion seems to be more appropriate. Let us call $\lambda \in \mathbb{K}$ an asymptotic eigenvalue of $L$ and $F$ if there exists an unbounded sequence $\left(x_{n}\right)_{n}$ such that

$$
\lim _{n \rightarrow \infty} \frac{\left\|\lambda L x_{n}-F\left(x_{n}\right)\right\|}{\left\|x_{n}\right\|}=0 .
$$

It is easy to see that the set of all asymptotic eigenvalues of $L$ and $F$ is then nothing else but the subspectrum $\sigma_{q}(L, F)$, and so belongs to the spectrum (16), by definition. With this notion of point spectrum, the following "discreteness result" is then parallel to the discreteness result for the spectrum of compact linear operators mentioned above. 
Theorem 4. Let $L$ be as before, and let $F$ be odd and compact. Then the inclusion

$$
\sigma(L, F) \backslash\{0\} \subseteq \sigma_{q}(L, F)
$$

is true, i.e. every non-zero spectral point is an asymptotic eigenvalue of $L$ and $F$.

Proof. Suppose that $\lambda \neq 0$ does not belong to $\sigma_{q}(L, F)$, i.e. $\left[\Phi_{\lambda}(L, F)\right]_{q}>0$. From Theorem $3 /\left(\right.$ a) we know then that $\lambda$ does not belong to $\sigma_{a}(L, F)$ either, so we have to show only that $\lambda \notin \sigma_{\delta}(L, F)$. By definition (15) and Lemma 1 (d), this is equivalent to proving that $\lambda L-F$ is stably solvable.

Let $G: X \rightarrow Y$ be compact with with $[G]_{Q}=0$. By assumption, $\lambda \neq 0$ and $\left[\Phi_{\lambda}(L, F)\right]_{q}>0$, and hence there exists $R_{1}>0$ and $\delta>0$ such that

$$
\left\|\lambda^{-1}(L+J P)^{-1}(\lambda L-F)(x)\right\| \geq \delta\|x\|
$$

whenever $\|x\| \geq R_{1}$. On the other hand, we can find $R_{2}>0$ such that

$$
\left\|\lambda^{-1}(L+J P)^{-1} G(x)\right\| \leq \frac{\delta}{2}\|x\|
$$

whenever $\|x\| \geq R_{2}$. Thus, for $\|x\| \geq R:=\max \left\{R_{1}, R_{2}\right\}$ and $0 \leq \mu \leq 1$ we have

$$
\left\|\lambda^{-1}(L+J P)^{-1}(\lambda L-F-\mu G)(x)\right\| \geq \frac{\delta}{2}\|x\| .
$$

Now,

$$
\lambda^{-1}(L+J P)^{-1}(\lambda L-F-\mu G)=(I-P)-\lambda^{-1}(L+J P)^{-1}(F+\mu G)
$$

is a compact perturbation of the identity. Therefore, from (22) and the homotopy invariance of the Leray-Schauder degree, we obtain for $\Omega_{R}$ being the open ball in $X$ with radius $R$

$$
\begin{aligned}
\operatorname{deg} & {\left[\lambda^{-1}(L+J P)^{-1}(\lambda L-F-G), \Omega_{R}, 0\right] } \\
& =\operatorname{deg}\left[\lambda^{-1}(L+J P)^{-1}(\lambda L-F), \Omega_{R}, 0\right] \\
& \equiv 1(\bmod 2),
\end{aligned}
$$

and so the equation $\lambda L x=F(x)+G(x)$ has a solution in $\Omega_{R}$

We remark that Theorem 4 may be generalized in the following way. If $F$ is odd and $[F]_{A}<\infty$, then every $\lambda \in \sigma(L, F)$ with $|\lambda|>[F]_{A}$ belongs to $\sigma_{q}(L, F)$, i.e. is an asymptotic eigenvalue of $L$ and $F$. This extends a corresponding result for linear operators due to Ambrosetti [2] which states that every spectral value $\lambda$ of a bounded linear operator $L$ with $|\lambda|>[L]_{A}$ is actually an eigenvalue of $L$.

At first glance, it seems more natural to call $\lambda$ an asymptotic eigenvalue of $L$ and $F$ if $(20)$ is replaced by the stronger condition

$$
\lim _{n \rightarrow \infty}\left\|\lambda L x_{n}-F\left(x_{n}\right)\right\|=0
$$

for some unbounded sequence $\left(x_{n}\right)_{n}$ in $X$. In fact, in the linear case (23) gives precisely the familiar definition of eigenvalue, while (20) does not. (To see this, consider $X=l_{2}$, $L=I, F\left(x_{1}, x_{2}, x_{3}, \ldots\right)=\left(x_{1}, \frac{1}{2} x_{2}, \frac{1}{3} x_{3}, \ldots\right)$ and $\lambda=0$.) However, definition (23) has also a flaw: Theorem 4 is no longer true with this definition of asymptotic eigenvalue. This is shown by the following example. 
Example 3. Let $X$ be an infinite dimensional real Banach space, $e \in X$ with $\|e\|=1$, and $\ell \in X^{*}$ a bounded linear functional on $X$ satisfying $\ell(e)=1$. Let $L=I$, and define $F: X \rightarrow X$ by

$$
F(x)=\frac{\|x\|}{1+\|x\|} \ell(x) e .
$$

Obviously, $F$ is odd and compact, and $[F]_{Q} \leq\|\ell\|$, and so $\sigma(I, F) \subseteq[-\|\ell\|,\|\ell\|]$.

Consider now the eigenvalue equation $F(x)=\lambda x$. Obviously, every non-trivial solution of this equation has the form $x=\mu e$, where $\lambda=\frac{|\mu|}{1+|\mu|}$, and so $0<\lambda<1$. But for fixed $\lambda \in(0,1)$ we can have only the two solutions $\mu= \pm \frac{\lambda}{1-\lambda}$ for $\mu$, and so (22) does not hold for any $\lambda$. On the other hand, choosing $x_{n}=n e$ we see that

$$
\frac{\left\|x_{n}-F\left(x_{n}\right)\right\|}{\left\|x_{n}\right\|}=\frac{\left\|n e-\frac{n}{1+n} n e\right\|}{n}=\frac{1}{1+n} \rightarrow 0 \quad(n \rightarrow \infty),
$$

and thus $1 \in \sigma_{q}(I, F) \subseteq \sigma(I, F)$.

We remark that Theorem 4 may be applied to existence theorems for boundary value problems involving nonlinear odd differential operators. Such applications will be given in a subsequent paper.

Acknowledgement. The authors express their gratitude to both referees for several interesting remarks which considerably helped to improve the paper.

\section{References}

[1] Akhmerov, R. R., Kamenskij, M. I. Potapov, Rodkina, A. E. and B. N. Sadovskij: Measures of Noncompactness and Condensing Operators (in Russian). Novosibirsk: Nauka 1986; Engl. transl.: Boston: Birkhäuser 1992.

[2] Ambrosetti, A.: Proprietà spettrali di certi operatori lineari non compatti. Rend. Sem. Mat. Univ. Padova 42 (1969), 189 - 200.

[3] Appell, J.: Implicit functions, nonlinear integral equations, and the measure of noncompactness of the superposition operator. J. Math. Anal. Appl. 83 (1981), $251-263$.

[4] Appell, J., De Pascale, E. and A. Vignoli: A comparison of different spectra for nonlinear operators. Nonlin. Anal. 40 (2000), $73-90$.

[5] Appell, J. and M. Dörfner: Some spectral theory for nonlinear operators. Nonlin. Anal. 28 (1997), 1955 - 1976.

[6] Appell, J., Giorgieri, E. and M. Väth: On a class of maps related to the Furi-MartelliVignoli spectrum. Annali Mat. Pura Appl. 179 (2001), 215 - 228.

[7] Deimling, K.: Nonlinear Functional Analysis. Berlin: Springer 1985.

[8] Feng, W.: A new spectral theory for nonlinear operators and its applications. Abstr. Appl. Anal. 2 (1997), 163 - 183.

[9] Feng, W. and J. R. L. Webb: A spectral theory for semilinear operators and its applications. In: Recent Trends in Nonlinear Analysis (ed.: J. Appell). Boston: Birkhäuser 2000, pp. $149-163$. 
[10] Furi, M.: The class of stably solvable operators is not stable under small perturbations (submitted).

[11] Furi, M., Martelli, M. and A. Vignoli: Stably solvable operators in Banach spaces. Atti Accad. Naz. Lincei Rend., Cl. Sci. Fis. Mat. Nat. 60 (1976), 21 - 26.

[12] Furi, M., Martelli, M. and A. Vignoli: Contributions to the spectral theory for nonlinear operators in Banach spaces. Annali Mat. Pura Appl. 118 (1978), 229 - 294.

[13] Gaines, R. E. and J. L. Mawhin: Coincidence Degree and Nonlinear Differential Equations. Lect. Notes Math. 568 (1977).

[14] Kachurovskij, R. I.: Regular points, spectrum and eigenfunctions of nonlinear operators (in Russian). Dokl. Akad. Nauk SSSR 188 (1969), 274 - 277; Engl. transl.: Soviet Math. Dokl. 10 (1969), $1101-1105$.

[15] Maddox, I. J. and A. W. Wickstead: The spectrum of uniformly Lipschitz mappings. Proc. Royal Irish Acad. 89-A (1989), 101 - 114.

[16] Mawhin, J. L.: Existence theorems for nonlinear operator equations and coincidence degree for some mappings in locally convex topological vector spaces. J. Diff. Equ. 12 (1972), $610-636$.

[17] Mawhin, J. L.: Topological Degree and Boundary Value Problems for Nonlinear Differential Equations. Louvain-la-Neuve (Belgium): Inst. Math. Pure Appl. Univ. Cath. Louvain 1991.

[18] Neuberger, J. W.: Existence of a spectrum for nonlinear transformations. Pacific J. Math. 31 (1969), 157 - 159.

[19] Pejsachowicz, P. and A. Vignoli: On the topological coincidence degree for perturbations of Fredholm operators. Boll. Unione Mat. Ital. 17-B (1980), 1457 - 1466.

[20] Rhodius, A.: Der numerische Wertebereich und die Lösbarkeit linearer und nichtlinearer Operatorgleichungen. Math. Nachr. 79 (1977), 343 - 360.

[21] Tarafdar, E. U. and H. B. Thompson: On the solvability of nonlinear noncompact operator equations. J. Austral. Math. Soc. 43 (1987), 103 - 114.

Received 11.12.2000; in revised form 05.06.2001 